\title{
UNA PROPUESTA QUE APUNTA A LA INTEGRACIÓN DE TRADUCCIÓN Y EMPRESA
}

\section{AL PROPOSAL TO INTEGRATE TRANSLATION AND BUSINESSES}

FECHA DE RECEPCIÓN: 14 de enero FECHA DE APROBACIÓN:10 de marzo Pp. 68-77

\section{Wilson Hernando García Trujillo*}

* Magister en Traducción Traductor Científico - Técnico y de Negocios / Interprete / Docente de Inglés de Negocios UEAN - U Pompeu Fabra Unimedios - Prensa - Universidad Nacional de Colombia 


\section{RESUMEN}

El presente artículo desarrolla la idea de la necesidad y la importancia de incluir un departamento de traducción profesional en los procesos de negociación de las empresas. El desarrollo de esta propuesta surge a partir de una investigación de carácter cualitativo que se realizó en la empresa TV3 LTDA. En el marco de esta investigación, el objeto de estudio está dado por los procesos de traducción que lleva a cabo la empresa. Los objetivos específicos incluyeron la identificación de los documentos que se requieren traducir al interior de la empresa y la caracterización de los procesos de traducción que se llevan a cabo dentro de la misma.

Con el fin de cumplir los objetivos, se utilizaron técnicas de recolección de información tales como: reunión de documentos, cuestionarios y entrevistas realizadas a todos los empleados de TV3 LTDA que habian enfrentado la necesidad de traducción. De esta manera, al llevar a cabo la investigación el resultado principal consistió en el planteamiento de una propuesta organizacional del servicio de traducción para TV3 LTDA, una propuesta ligada específicamente a las necesidades de la empresa y sujeta a los parámetros de calidad de la Norma Europea De Calidad CEN 15038 de la ISO.

\section{ABSTRACT}

This article develops the idea of the necessity and importance of including a professional translation department in the negotiation process of enterprises. This proposal emerges from a qualitative research performed at TV3 LTDA. Within the frame of this research, the object of study is composed by the translation processes carried out in the enterprise. The specific objectives included the identification of the documents to be translated and the characterization of the translations processes implemented within the enterprise.

For accomplishing the objectives previously mentioned, different data collection techniques were used, such as document gathering, questionnaires and interviews performed to all TV3 employees involved in translation processes. The main outcome of this research was the generation of an organizational proposal for the translation services in TV3 LTDA, a proposal specifically linked to the enterprise's needs and subject to the quality parameters of the CEN-15038 European Quality Standards for

\section{Palabras claves}

Empresa

Globalización

Traducción

Traductor

Beneficio económico

Participación

Cambio

Estandarización

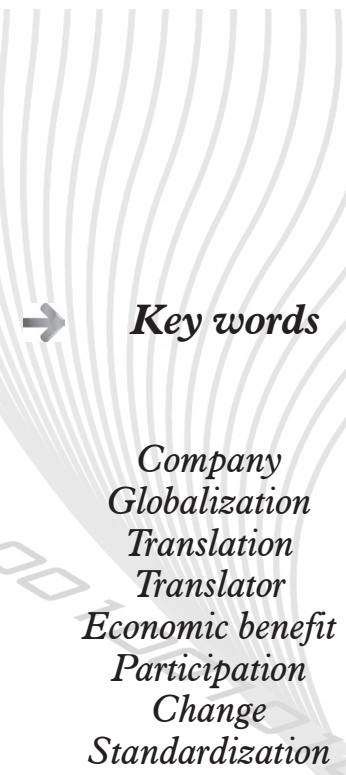

\section{RESUMÉÉ}

Translation Services

Cet article traite de la nécessité et de l'importance d'inclure un service de traduction professionnel lors du processus de négociation d'affaires et autres contrats. Le développement de cette proposition découle d'une recherche qualitative menée chez TV3 Ltda. Dans le cadre de cette enquête, l'objet d'étude est dévoilé par les processus de traduction réalisée par la société. Les objectifs spécifiques visaient l'identification des documents dont la traduction était nécessaire au sein de l'entreprise et la caractérisation des processus de traduction étant menée au sein de cette dernière. Afin de remplir les objectifs mentionnés précédemment, des techniques de collecte de renseignements ont été utilisées tels que la réunion de documents, la création de questionnaires et la mise en place d'entretiens avec tous les employés de TV3 LTDA ayant fait face, à au moins une reprise, à la nécessité de la traduction. De cette façon, le résultat principal des recherches menées a été une nouvelle proposition d'organisation du service de traduction de TV3 LTDA, proposition liée aux besoins spécifiques de l'entreprise et sujette aux paramètres de qualité de la Norme Européenne de qualité ISO CEN 15038. 


\section{INTRODUCción}

E sta investigación se desarrolló con el fin de presentar la tesis de grado para la Maestría en traducción de negocios de la Universidad EAN. A partir de un estudio de caso realizado a la empresa TV3 LTDA, la cual se desempeña en el área de la publicidad, se observaron las características de los procesos de traducción que lleva a cabo dicha empresa. Mediante este estudio se logró establecer que en un mundo empresarial, dentro de un sector que demanda apertura al cambio permanente, en un contexto actual que tiende a las innovaciones aceleradas, es necesario incluir un traductor profesional en los procesos de negociación de las empresas. Esto con el fin de optimizar los procesos internos de traducción que la empresa requiera para así obtener utilidades en menor tiempo y mayores beneficios en términos económicos. Esto no solo favorecería a la empresa como tal, haciendo sus procesos de negociación y traducción internacional más eficaces, sino que beneficiaría al traductor posicionándolo en el mundo empresarial como una figura dinámica y participativa en los procesos internos de la empresa.

La propuesta que surge consiste en incluir un departamento propio de especialistas en traducción y terminólogos en la empresa, de tal manera que esta pueda contar constantemente con un servicio que no es ajeno a si misma, sino que hace parte de sus procesos empresariales, capaz de trabajar al unísono, en una misma dirección, teniendo en cuenta una dinámica equivalente y siguiendo un mismo propósito.

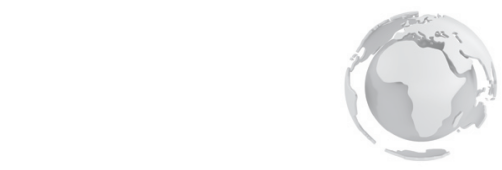

\section{METOdología}

(2) e realizó una investigación descriptiva de carácter cualitativo en la que se especificaron propiedades, características, perfiles, grupos, personas y fenómenos que intervienen en el proceso de traducción al interior de TV3 LTDA. La investigación identificó las necesidades de traducción con las que posteriormente se generó la propuesta de servicios de un sistema organizacional con base en los elementos con los que cuenta la empresa.
Los instrumentos utilizados para la recolección de información consistieron en reunir los documentos que la empresa necesita traducir, se realizaron entrevistas a los miembros de la empresa que alguna vez se han enfrentado a un proceso de traducción dentro de la empresa, así mimo se aplicaron encuestas a dichos miembros. 


\section{GLOBALIZACIÓN Y TRADUCCIÓN}

na de las más grandes y evidentes características del fenómeno de la globalización es la necesidad de procesos comunicativos rápidos y eficaces, esta necesidad

ha generado que se busque una

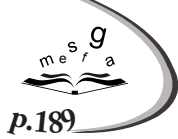

lengua estándar, como es bien sabido, esta lengua es el inglés. El inglés es

el vehículo lingüístico del mundo globalizado y actúa como ícono de lo moderno e innovador (Montes,2007).

Traduccion y Globalizacion. Analisis y Perspectivas del Fenómeno Publicitario.

Frente al fenómeno de la globalización cabe analizar dos aspectos que determinan la relación de la empresa con esta: el inglés como lingua franca ${ }^{1}$ y la importancia de la estandarización ${ }^{2}$. El mundo globalizado exige aprovechamiento máximo del tiempo y del espacio, en la actualidad el mundo es considerado una aldea global en la que el tiempo y el espacio quedan comprimidos en nanosegundos, (Montes,2007). Traduccion y Globalización. Analisis y Perspectivas del Fenómeno Publicitario. En este sentido es posible afirmar que una empresa al carecer de un proceso estándar en la fase de traducción, dentro de los procesos de negociación, se encuentra en desventaja en cuanto a la no optimización del tiempo en términos de contratación y las pocas posibilidades que tiene de incursionar en países de habla inglesa.

TV3 LTDA aunque se ha posicionado dentro del mercado local y es una empresa exitosa locamente, podría aprovechar esta reputación y adicionar un traductor que no solo se encargue de la traducción de la documentación corporativa, sino que lleve a la compañía a través de la totalidad del proceso de negociación internacional para, de esta manera, ingresar a mercados potenciales en países que utilicen el inglés como medio de comunicación. Consecuentemente, la empresa no solo mejoraría sus ingresos, sino que tendría una visión cosmopolita y multicultural coherente con el mundo globalizado. "El mundo globalizado se define por una visión cosmopolita y multicultural de las sociedades. El intercambio de las nuevas tecnologías de la información y la comunicación se articula mediante una lengua, el inglés, que ha adquirido el estatus de lingua franca en las relaciones internacionales". (Montes, 2007). Traduccion y Globalizacion. Analisis y Perspectivas del Fenómeno Publicitario.

En cuando al factor estandarización, "La publicidad global se convierte en un poderoso instrumento para difundir los modelos y los estilos de vida que se popularizan en todo el mundo" (Montes, 2007). Dado que TV3 Ltda., es una empresa de publicidad que se encarga de la difusión de ideas y que claramente necesita convencer al cliente de que consuma algún producto, es importante que tenga en cuenta las tendencias actuales, que específicamente se pueden resumir en la negación de la diversidad, es decir, la estandarización es el objetivo de la globalización y del pensamiento global. "la estandarización es una campaña publicitaria global" (Montes, 2007, p. 5). En este momento, la estandarización esta dictada por los países de habla inglesa, en especial Estados Unidos, de esta manera TV3 LTDA puede restringir sus posibilidades de comprender el comportamiento global del mercado y de la publicidad desde la relación que existe entre lengua y cultura. En este sentido, Montes afirma que "la publicidad es un fiel reflejo de las convicciones y normas, los ideales y modelos. La publicidad traspasa todas las fronteras, al utilizar el mismo mensaje publicitario en una variedad de culturas. A través de campañas publicitarias internacionales basadas en marcas globales, se difunden unos valores universales que vienen sustentados, en su mayoría, por la cultura anglo americana (...) debido a que los productos y las marcas provienen en su mayoría de empresas norteamericanas". (Montes, 2007).

De esta manera "observamos que el consumo a nivel globalizado ha suscitado determinados modelos de

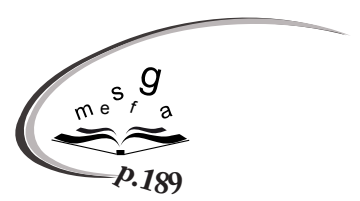

\footnotetext{
1 Término acuñado por Montes (2007).

${ }^{2}$ Término acuñado por Montes (2007).
} 
comportamiento en los cuales coinciden diferentes grupos de consumidores de diversos países". (Montes, 2007). Así se llega a la conclusión de que es indispensable consolidar una estrategia que le permita a TV3 LTDA posicionarse en el mundo globalizado mediante la adopción del manejo de la lengua inglesa y el pensamiento globalizado en sus procesos internos y en su proyección internacional incluyendo a traductor especialista dentro de sus procesos de negociación y traducción y consolidando un departamento propio que se encargue de dichos procesos.

\section{NECESIDAD DE POTENCIAR Y POSICIONAR AL TRADUCTOR}

ebido al fenómeno de globalización que ha experimentado el mundo en los últimos años, la demanda de servicios lingüísticos, concretamente de traducciones, se ha incrementado de forma exponencial, lo cual ha obligado tanto a traductores como a empresas de traduc-

ción a transformar radicalmente su perfil, lo que también ha conllevado a una mayor participación de un sector en expansión. "La traducción ha dejado de ser un producto de consumo minoritario

para convertirse en parte de la cadena de fabricación de productos o de suministro de servicios, con todas las ventajas e inconvenientes que ello conlleva. (Zaragoza, 2006).

Sin embargo, el mismo fenómeno de la globalización y el hecho de que el mercado de la traducción sea tan necesario y este creciendo a paso acelerado, presenta una serie de consecuencias que afectan negativamente la calidad de la prestación del servicio, así como a los que lo solicitan. Entre otras las situaciones que se presentan con relación al servicio de traducción son la prestación del servicio de traducción a muy bajos precios y de baja calidad, la utilización de personal no calificado para dicha labor y el desconocimiento por parte de los clientes a cerca del producto por el cual están pagado. "El descenso de precios es el resultado de la prestación de servicios de traducción por parte de empresas o individuos no profesionales (academias, empresas de azafatas y congresos, consultorías, extranjeros que llegan al país pero que no tienen formación específica, nacionales que han vivido fuera pero que no tienen formación específica, personal interno de las empresas, etc.) o por parte de empresas o entidades, incluso públicas, que se valen de malas prácticas empresariales para enriquecerse rápidamente (morosidad, utilización de becarios para ofrecer servicios profesionales, reducción de costos y de precio a base de la

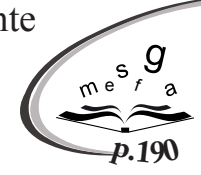
eliminación de infraestructura y de controles internos de calidad, concursos a precios por debajo de mercado, etc.). A esto se le llama dumping de precios y es un fenómeno que está presente en todos los sectores y que en el de la traducción es un elemento distorsionador de la competencia muy grave.

Prácticamente la mitad de las empresas cubre sus necesidades de traducción mediante personal interno no especializado. Este dato nos revela uno de los problemas principales a los que se enfrenta el sector de la traducción: el cliente no valora en su justa medida la importancia de una traducción de calidad. Es más, muchas veces no sabe qué es una traducción de calidad porque desconoce el producto, la profesión y el proceso de producción y porque carece de los conocimientos (de idiomas) necesarios para valorar la calidad del producto (traducción) que acaba de comprar". (Zaragoza, 2006).

En el caso especifico de TV 3 LTDA, mediante la realización de la investigación se logró evidenciar la carencia de un departamento propio de traducción. Según 
la información proporcionada por la empresa, se identificó que en las ocasiones en que la empresa requiere de la traducción de algún documento, se sirve de un traductor externo, que no siempre es el mismo. Así mismo se evidenció que la empresa ha perdido oportunidades de hacer negociaciones internacionales debido a la falta de un departamento o profesional que se encargue de hacer este tipo de negociaciones.
Esta situación justifica y sustenta la necesidad de establecer un departamento de traducción dentro de la empresa de tal manera que el traductor se involucre de manera activa dándole valor agregado a la empresa y posicionándose en el medio empresarial a partir de sus conocimientos lingüísticos.

\section{BENEFICIOS ECONÓMICOS PARA LAS EMPRESAS}

E studiar la manera en que la traducción optimiza, o en su defecto, obstaculizaba los procesos normales de funcionamiento y mercado de una empresa, es de suma importancia en tanto se pretende proponer un proyecto que este ligado directamente con la empresa relacionado con sus procesos de traducción de documentos y negociaciones internacionales.

Como se mencionó anteriormente, y con el fin de presentar un ejemplo concreto, basado en la investigación realizada, cada vez que TV3 LTDA se enfrenta a un proceso de contratación para realizar traducciones de documentos o negociaciones en lengua inglesa, ésta lleva a cabo el proceso desde el principio debido a la falta de una base de datos que posibilite recurrir a los servicios de los que se han beneficiado anteriormente. De esta manera se emplea más tiempo del necesario y a la empresa se le incrementan los costos, además de correr el riesgo de que los servicios de traducción que se contraten no correspondan a las necesidades de la empresa, es evidente que con este proceso la compañía esta perdiendo no solo tiempo sino también utilidades.

De esta manera es evidente que, si la empresa cuenta con, no solo un departamento de traducción, sino con una base de datos terminológicos y un servicio constante de apoyo, puede reducir el riesgo de obtener traducciones de baja de calidad o perder contratos internacionales por falta de personal calificado.
Tomando en cuenta lo anterior, terminología y traducción se complementan para ofrecer al sector empresarial la posibilidad de generar mayores ingresos y productividad partiendo de la incorporación del traductor especializado y el terminólogo dentro de la dinámica empresarial, esto por su parte proporcionará beneficios para el desarrollo de los dos sectores de manera simultánea. Surge como una necesidad más que como un requisito debido al carácter ambivalente del lenguaje y la importancia de la precisión. "Trabajar con dos lenguas significa que las palabras no se corresponden exactamente de una lengua a otra, por más que sus definiciones concuerden, puesto que la realidad que las rodea y los contextos en que se producen no se segmentan de la misma manera" (Rodríguez, 2004).

En este sentido, es pertinente mencionar el valor agregado que le proporciona un traductor especializado, o un departamento especializado en traducción a una empresa, en el sector empresarial una traducción que corresponda a un discurso especializado y especifico, con un uso adecuado de la terminología correspondiente a la disciplina conlleva a un incremento en la claridad, en el propósito de los documentos, ahorra tiempo y dinero, aumenta la calidad del producto y por tanto el prestigio de la empresa. Además de acrecentar la satisfacción del cliente, disminuir el tiempo de comercio, fortalecer la imagen corporativa, crear bases de datos útiles a largo plazo y aumentar la autonomía corporativa. Esto, a partir 


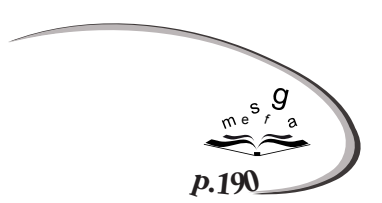

de la toma de medidas que aseguren que el contenido de los términos usados en una compañía, sean exactos, apropiados y coherentes (corporate terminology).

Es indispensable determinar cuáles deben ser los criterios, las aptitudes y las posibilidades de actuación del traductor especializado dentro del sector empresarial. De esta manera, por su parte, el traductor debe cumplir ciertos criterios que lo califiquen con el fin de mejorar los procesos internos que le competan dentro del marco empresarial, así, su desempeño proporcionará los beneficios ya mencionados y su permanencia dentro de los procesos empresariales será cada vez más necesaria.

Es preciso entonces que el traductor tenga una amplia competencia cognitiva, entendida como el conocimiento suficiente del ámbito especializado, además una desarrollada competencia lingüística que constituye el dominio de las dos lenguas, debido a que, en la traducción especializada se presentan unidades específicas pertenecientes a un ámbito especializado; por lo tanto al traductor le corresponde no solo saber dichas unidades en el texto original, sino saber encontrarles el equivalente adecuado en la lengua de llegada (Rodríguez 2004) y por último, una competencia comunicativa que le facilitará comunicar el conocimiento especializado y le otorgará las herramientas necesarias para conseguir una comunicación eficaz.
En este sentido, el traductor especialista en el ámbito empresarial debe ser ante todo un investigador enfocado principalmente en el trabajo terminológico, en la búsqueda constante de precisión, transparencia, consistencia y economía lingüística. Esto con el fin de establecer sistemas de conceptos que contribuirán al desarrollo y al avance de la especificidad lingüística y empresarial. Así, el sector empresarial puede obtener suficientes ventajas por la inclusión del traductor especializado, el cual, además de prestar servicios de terminología y traducción para las compañías, puede proporcionar, servicio de escritores técnicos, soluciones para negocios (sitios web), redacción técnica terminológica, redacción monolingüe y recuperación de información, contribuyendo así al incremento de la claridad de la información que circula al interior de la empresa y corrigiendo oportunamente, si es el caso, errores o terminología polivalente que pueda traer consecuencias negativas a futuro.

Con el fin de incluiral traductor especialista en las empresas, ya no como una unidad colaboradora en los procesos productivos, sino cómo un eje generador de conocimiento y capacidad de dinamizar procesos al interior de la empresa, es indispensable que el profesional de lenguaje promueva su participación a través de la creación de redes sociales que posibiliten flujos de trabajo, expansión y conocimiento del mercado, además de presentar en sus currículos su especialidad lingüística y terminológica para que las empresas vean en la profesionalización del oficio un valor agregado y que, por su parte, las empresas promuevan un modelo de contratación "in house" para que la subcontratación se desvirtúe paulatinamente y tanto el sector empresarial como el traductor especialista se vean beneficiados.

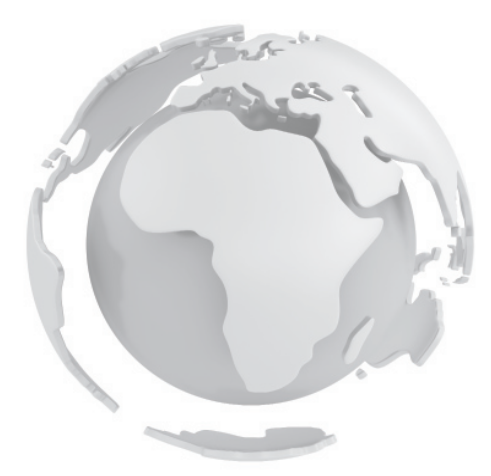


partir de las observaciones al proceso de traducción que TV3 Ltda., realiza y la información bibliográfica consultada se pudo evidenciar que al optimizar los procesos de traducción y gestión de la traducción de la manera en que se expone en la propuesta que se planteó, la compañía ahorrará tiempo y recursos, al evitar que dichos procesos se lleven a cabo de manera siempre diferente y sin garantía de la consecución de un producto de calidad y que satisfaga la necesidad que la empresa tiene. De esta manera se reduce drásticamente la posibilidad de que TV3 Ltda., tenga problemas legales, comerciales y financieros debido a prácticas inadecuadas en sus procesos de traducción y negociación.

Así mismo, se evidenció, a partir de la observación realizada al proceso de traducción que lleva a cabo la empresa, que es necesario que el traductor salvaguarde la disciplina y no caiga en el círculo que Montes denomina dumping de precios, el cual se da gracias a la interacción de tres factores: una gran demanda del servicio, una alta competencia y la necesidad de las empresas de reducir costos. Estos factores son mencionados por Montes con el fin de argumentar que el fenómeno genera prácticas inadecuadas en el campo de la traducción. En TV3 Ltda., es evidente que el fenómeno mencionado se presenta en sus procesos de traducción; es decir, de acuerdo con lo observado durante la investigación, la empresa acepta ofertas de traducción de las que no se tiene conocimiento previo del trabajo que se realiza. Por esta razón es difícil verificar su nivel de calidad y aunque en apariencia resuelve la necesidad inmediata y parece reducir costos, se observó que a largo plazo aumenta los costos de este servicio.
Finalmente, ya que se tomó como referente de observación una empresa dedicada a la publicidad, se puede decir que existen diferentes aspectos socioculturales y socioeconómicos que se dan a partir de la traducción relacionada con este campo. En este sentido la práctica de la traducción debe ser, no sólo suficientemente responsable con la información que esté a su cargo, sino que también debe tener conocimientos socioculturales suficientes que permitan establecer un diálogo coherente entre la información a traducir y la información traducida. De esta manera, es posible relacionar más a fondo la traducción y la publicidad con el escenario de un mundo que camina a pasos acelerados hacia la globalización, ya que esta es un reflejo de la forma cultural, sociológica y económica que domina las relaciones y formas de comunicación. En efecto no se puede analizar relación traducción-publicidad aislada sin tener en cuenta el espacio sociocultural y socioeconómico en las que estas dos están inmersas. Bajo esta mirada y teniendo en cuenta la propuesta de servicio que se propuso, con el fin de optimizar los procesos de traducción de la empresa, se puede afirmar que TV3 Ltda., podría posicionarse en un mundo globalizado y adquirir más potencial de competitividad en el caso en el que incorporara dentro de sus procesos de funcionamiento la propuesta desarrollada en este proyecto.

Los resultados obtenidos de esta investigación son de gran alcance, tanto para el sector empresarial, como para el campo de la traducción en tanto surge de una manera explicita un beneficio mancomunado, es decir, se beneficia la empresa gracias a la optimización de sus procesos de traducción y negociación en lengua extranjera y se beneficia el campo de la traducción al acercarse y trabajar de manera interdisciplinar con el sector empresarial. 
1 ara concluir se puede afirmar que la implicación más sobresaliente de esta investigación en el sector empresarial y en el campo de la traducción es la promoción en las empresas de procesos de estandarización, lo cual, es en sí mismo un sinónimo de eficiencia. Es decir, con la aplicación de la propuesta, que está basada en la norma ISO se mejora la eficiencia de una compañía en la misma proporción en la que avanza su capacidad de estandarizar procesos y así, indudablemente, se aumenta la productividad y las ganancias. En este caso específicamente se habla de estandarización desde el punto de vista de la globalización, es decir, una propuesta como la presentada, que consiste en incluir un departamento de traducción dentro de los procesos internos de la empresa, con el fin de que la traducción de documentos y las negociaciones internacionales dejen de ser un proceso aislado de la empresa en si misma, apunta a estar a la vanguardia en una época de globalización e integración de los campos del conocimiento.

La urgencia de la estandarización es un fenómeno que incluye a todos los sectores sociales y en consecuencia al lenguaje mismo. Las múltiples manifestaciones por las que hoy en día se nos revela la realidad, no son otra cosa que una posibilidad más de mirar la totalidad de las disciplinas cómo una integración del conocimiento sin ninguna alternativa de fragmentación. La fusión entre el lingüista y el sector empresarial no son más que una confirmación de este hecho.

Es responsabilidad de los traductores especialistas y terminólogos promover la profesionalización de la práctica para dejar de lado la idea de que la traducción se puede ejercer de manera informal, únicamente porque se ha garantizado el conocimiento de dos lenguas o más. Además el traductor especialista se encuentra en obligación de poseer el espíritu emprendedor para originar un cambio, no solo en la mentalidad del cliente o de las empresas, sino también de los propios traductores.

La diseminación de la profesionalización se debe llevar a cabo en cada práctica de traducción y terminología que el traductor acepte promoviendo entre sus clientes la visión de la traducción una posibilidad que mejora los procesos empresariales y fomentar la necesidad de un departamento que se dedique a este fin. Además de reiterar entre sus colegas la importancia de capacitarse y profesionalizarse "el papel de las universidades formadoras de traductores es desarrollar competencias en gestión y emprendimiento, que unidas a las competencias traductoras, permitan hacer que el sector de servicios de la traducción en Colombia tenga mayor visión de empresa y se constituya en un gremio formado por profesionales empresarios" (Clavijo p.35).

El futuro de la traducción y la terminología se dirigen hacia las empresas, a su vez el futuro de la empresa se fusiona cada vez más con el del traductor. El traductor especializado y su conocimiento pueden incidir significativamente en el crecimiento empresarial en tanto aporta eficacia en los diferentes procesos que se dan dentro de esta.

En cuanto a los objetivos que se plantearon al iniciar la investigación se puede afirmar que en general fueron alcanzados en su totalidad, es decir, se identificaron las necesidades y el proceso de traducción al interior de una empresa perteneciente al sector de la publicidad: TV3 Ltda. Además se contrastó dicho proceso con los procesos estándar (norma de calidad CEN 15038 de la ISO) con el fin de generar una oferta de traducción que optimizara lo que ya se venia haciendo con relación a este aspecto al interior de TV3 Ltda. Al contrastar el proceso actual de traducción en TV3 Ltda., con el proceso estándar recomendado por la norma CEN 15038 de la ISO. se generó una propuesta organizacional del servicio de traducción para TV3 Ltda., a partir de las necesidades específicas de la empresa, esto con el fin de generar valor agregado a la empresa y proporcionar soluciones viables que den solución a dicha necesidad.

Hasta el momento se planteó la propuesta y se le presentó a la empresa como una posibilidad para los procesos de traducción de la misma, en un futuro se espera tener conocimiento de los resultados y de los beneficios concretos que se presenten a partir de la aplicación de la propuesta proporcionada por el desarrollo de esta investigación, así mismo se espera que esta propuesta sea aplicable en un futuro en otras empresas, no solo pertenecientes al sector de la publicidad sino a los diferentes sectores empresariales. 


\section{REFERENCIAS BIBLIOGRÁFICAS}

Clavijo, S. et al. (2008) Babel en las Empresas Colombianas: Una mirada actual de la traducción. Universidad EAN. 2008.

Montes, A. (2007). Traduccion y Globalizacion. Analisis y Perspectivas del Fenómeno Publicitario (Inglés, Español, Alemán). Editorial Comares. Interlingua. España.

Rodríguez Camacho, E. (2004) Terminología y traducción: Gestión de la terminología en la traducción de textos especializados. Universidad del valle. 2004.

Zaragoza, J. (2006). La futura Norma de Calidad para los servicios de traducción. En: http://www.slideshare.net/ Tradeus/norma-europea-de-calidad-en-15038

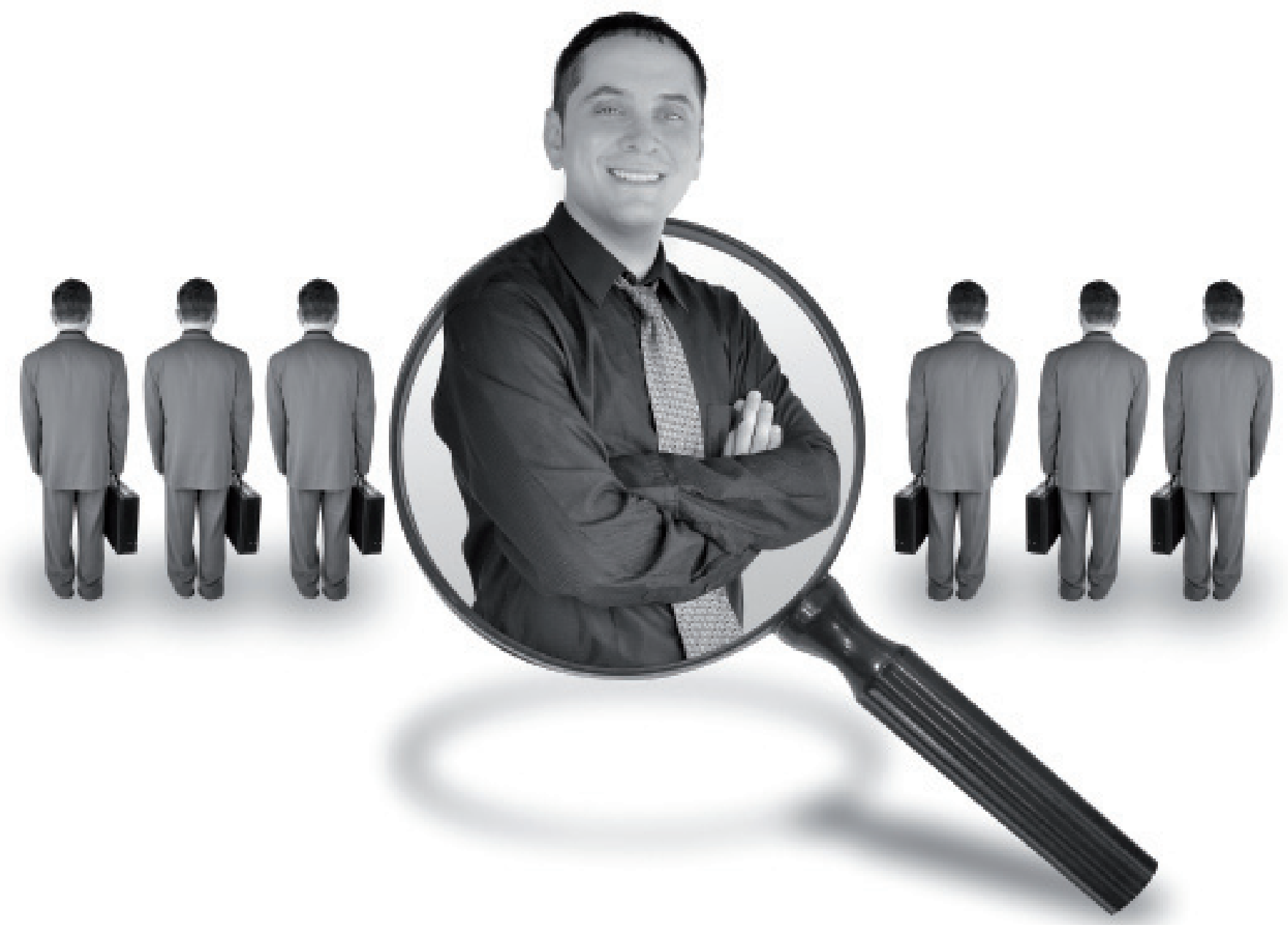

